\title{
Índice de Controle Ambiental das Unidades de Conservação do Maranhão
}

\author{
Environmental Control Index of the Protect Areas of Maranhão
}

\author{
Yata Anderson Gonzaga Masullo, Helen da Costa Gurgel ${ }^{2}$, Anne-Elizabeth Laques ${ }^{3}$, Dionatan Silva Carvalho ${ }^{4}$, Claudio \\ Eduardo de Castro 5
}

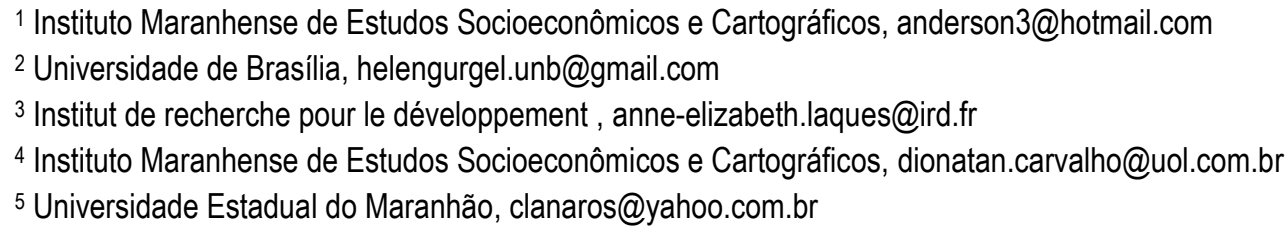

Recebido (Received): 11/10/2017 Aceito (Accepted): 01/10/2018

Resumo: O presente estudo objetiva expor o desenvolvimento do Índice de Controle Ambiental - ICA das Unidades de Conservação do Maranhão, a partir da mensuração de indicadores ambientais e institucionais. Busca-se dessa forma, representar a influência da dinâmica territorial sobre a efetividade das 11 UCs do Maranhão. Metodologias como esta tornam-se cada vez mais indispensável, em um cenário de institucionalização de área protegidas e efetivação de parques outrora somente existentes em papel. Avançase nesse contexto, a partir da proposição e desenvolvimento de métodos capazes de considerar as complexidades envolvida na questão, como a relação entre a escala local e regional, rápida aplicação, alta capacidade de reprodução e baixa subjetividade. Para o desenvolvimento da pesquisa, utilizou-se o método de análise multicritério conhecido como Analytic Hierarchy Process (AHP), alicerçados por técnicas ligados ao Sistema de Informação Geográfico - SIGs. Desse modo foi possível identificar que entre as UCs em análise, $18 \%$ possuem nível insatisfatório de efetividade, enquanto que outros $18 \%$ possuem grau pouco satisfatório, $54 \%$ possui nível medianamente e $18 \%$ nível reconhecido como satisfatório. A consolidação desta metodologia traz consigo contribuições aplicadas a otimização do planejamento, implantação e gestão das Unidades de Conservação do Maranhão, bem como em outros estados, a partir deste estudo.

Palavras-chave: Indicadores; Análise Multicritérios; AHP; SIG; Áreas Protegidas.

Abstract: The present study aims to develop the Environmental Control Index - ECI (ICA, in portuguese) of the protect areas of Maranhão, based on the measurement of environmental and institutional indicators. This research seeks to represent the influence of territorial dynamics on the effectiveness of protected areas applied in 11 PAs of Maranhão. Methodologies such as the aforementioned become increasingly indispensable, in a scenario of institutionalization of conservation units and implementation of paper parks. It advances in this context, from the proposition and development of methods capable of considering the complexities of protected areas, such as the relation between local and regional scale, rapid application, high reproductive capacity and low subjectivity. For the development of the research, we used the multicriteria analysis method known as Analytic Hierarchy Process (AHP), based on techniques linked to the Geographic Information System (GIS). In this way it was possible to identify that 18\% of the PAs under analysis have an unsatisfactory level of effectiveness, while 18\% have an unsatisfactory degree, 54\% have a medium level and 18\% have a satisfactory level. The consolidation of this methodology brings with it applied contributions to the optimization of planning, implementation and management of the protect areas of Maranhão, as well as in other locations, from this study.

Keywords: Indicators; Multicriteria Analysis; AHP; GIS; Protected Areas. 


\section{Introdução}

De acordo com a União Internacional para a Conservação da Natureza-UICN, as áreas protegidas - AP são reconhecidas como partes do território com limites geográficos definidos e reconhecidos, cujo intuito é a conservação da natureza (MCNELLY et al., 2004). Atualmente, essa estratégia de conservação da biodiversidade e do patrimônio cultural se multiplicou, alcançando aproximadamente 238 mil áreas protegidas designadas em 244 países, abrangendo aproximadamente $14,9 \%$ da superfície terrestre e 7,3\% da área oceânica global (UNEP; WCMC; UICN, 2018). No entanto, não podemos considerar somente a cobertura das APs como uma medida de eficácia ou mesmo do sucesso da conservação, faz-se necessário considerar o contexto, nível de representatividade e concentividade dessas áreas. Corroborando com essa perspectiva, Leverington et al. (2010), demonstram que somente $24 \%$ das áreas protegidas do mundo possuem efetividade satisfatória, enquanto que em $36 \%$ a gestão é somente regular, $27 \%$ possuem grandes deficiências de gestão e $13 \%$ apresentam nível de efetividade inadequada.

Originário desse contexto, o presente estudo possui o objetivo de desenvolver o Índice de Controle Ambiental aplicado às Unidades de Conservação-UC do Maranhão. Visa-se desse modo avaliar os tipos de usos e se a estrutura de governança da UC é adequada ao nível de proteção da área protegida e como influenciam o nível de efetividade dessas áreas protegidas. Para tanto, a presente pesquisa estrutura-se a partir da descrição dos procedimentos metodológicos de análise multicritérios, Analytic Hierarchy Process AHP, auxiliados por técnicas ligadas ao Sistema de Informação Geográfico - SIG. A partir da sintetização de indicadores ambientais e institucionais, avalia-se o nível de conservação e alteração da paisagem das UCs em estudo, identificando os mecanismos institucionais e os instrumentos de gestão existentes nelas, bem como analisa-se os avanços, limitações e a capacidade deste modelo metodológico em ser empregado diretamente no estudo da efetividade em áreas protegidas. Busca-se assim oferecer contribuições aplicadas a otimização do planejamento, implantação e gestão das Unidades de Conservação no estado do Maranhão.

\section{Materiais e Métodos}

Para o desenvolvimento do estudo, foram selecionadas 11 UCs do Maranhão e suas respectivas zonas de amortecimento. Divididas entre as categorias de Proteção Integral e de Uso Sustentável, as UCs do Estado são distribuídas entre 3 UCs Estaduais com características de proteção integral e 8 UCs Federais, três delas de proteção integral e cinco de uso sustentável (Quadro 1; Figura 1). Essas UCs foram criadas com a finalidade de proteger regiões de grande relevância socioambiental e cultural, tanto do bioma Amazônico quanto do Cerrado, abarcando atualmente 21 municípios e uma área de pouco mais de $13.809 \mathrm{~km}^{2}$, que corresponde a $4,2 \%$ do território estadual.

A proposta metodológica do estudo desenvolve-se sob uma abordagem sistêmica e multidisciplinar (MORIN et al., 2003). Considera-se para tanto a análise de variáveis primárias e secundárias selecionadas com base na sua disponibilidade, confiabilidade e periodicidade de atualização dos dados, estando estes interligados aos elementos globais de efetividade, conforme Leverington et al. (2010) e Schulze et al. (2017).

Com o intuito de apresentar a aplicação dos procedimentos metodológicos, para obtenção de resultados mensuráveis relativos ao estudo, faz-se necessário delinear-se as etapas de seleção, coleta, processamento, validação, modelagem e análise dos dados, estruturados aqui em 3 etapas:

Etapa 1: Inicialmente, a fim de se estabelecer um padrão de conformidade em todos os procedimentos técnicos da pesquisa, definiu-se os indicadores que fundamentaram o estudo. Estes indicadores foram selecionados através da realização de revisão bibliográfica sobre a temática (MACKINNON et al., 1990; PADOVAN, 2001; ERVIN, 2003; STOLTON et al., 2007; WWF, 2009; MARINELLI, 2011 e ICMBIO/WWF, 2017), posteriormente validados juntamente com gestores das UCs, representantes dos conselhos participativos (Meio Ambiente e Cidades) e pesquisadores do Instituto Maranhense de Estudos Socioeconômicos e Cartográficos - IMESC e Universidade Estadual do Maranhão - UEMA. Esses foram divididos em duas dimensões, com base em Padovan (2001) e Stoll-Kleemann (2010): Dimensão institucional se refere à capacidade de governança para lidar com os problemas e desafios ambientais e sociais, a partir dos instrumentos de gestão disponíveis; Dimensão ambiental refere-se ao nível de conservação e alteração da paisagem;

Em relação aos dados secundários, estes foram selecionados de acordo com a disponibilização de estatísticas existentes em nível de município e setor censitário, correspondendo a área das UCs em estudo. Utilizou-se o ano de 2010 como base, considerando a disponibilidade das demais variáveis. Ressalta-se que as variáveis selecionadas, foram cedidas por meio de instituições públicas e de pesquisa como o Instituto 
Chico Mendes de Conservação da Biodiversidade - ICMBio, IMESC, Secretaria Estadual de Meio Ambiente - SEMA e extraídos de imagens de satélite.

Quadro 1: Caracterização das Unidade de Conservação em estudo.

\begin{tabular}{|c|c|c|c|c|c|}
\hline Unidade de Conservação & Criação & $\begin{array}{l}\text { Nível de } \\
\text { Proteção }\end{array}$ & Bioma & $\begin{array}{l}\text { Área } \\
\left(\mathrm{Km}^{2}\right)\end{array}$ & Municípios (MA) \\
\hline $\begin{array}{c}\text { Parque Estadual do } \\
\text { Bacanga }\end{array}$ & $\begin{array}{l}\text { Lei Estadual } \mathrm{N}^{\circ} 7.545 \mathrm{de} \\
02 / 03 / 1980\end{array}$ & Proteção Integral & Amazônia & 26 & São Luís \\
\hline $\begin{array}{l}\text { Parque Estadual do } \\
\text { Mirador }\end{array}$ & $\begin{array}{l}\text { Lei Estadual } \mathrm{N}^{\circ} 7.641 \mathrm{de} \\
04 / 06 / 1980, \text { alterado pela Lei } \\
\text { Estadual } \mathrm{n}^{\circ} 8.958 \mathrm{de} \\
08 / 05 / 2009\end{array}$ & Proteção Integral & Cerrado & 4.370 & Mirador, Formosa da Serra Negra \\
\hline $\begin{array}{l}\text { Parque Estadual do Sítio } \\
\text { do Rangedor }\end{array}$ & $\begin{array}{l}\text { Lei Estadual } \mathrm{N}^{\circ} 21.797 \mathrm{de} \\
15 / 12 / 2005 \text {, alterado pela Lei } \\
\text { Estadual } \mathrm{N}^{\circ} 10.455 / 2016\end{array}$ & Proteção Integral & Amazônia & 1,3 & São Luís \\
\hline $\begin{array}{l}\text { Parque Nacional dos } \\
\text { Lençóis Maranhenses }\end{array}$ & $\begin{array}{l}\text { Lei Federal } \mathrm{N}^{\circ} 86.060 \mathrm{de} \\
02 / 06 / 1981\end{array}$ & Proteção Integral & Cerrado & 1.550 & $\begin{array}{c}\text { Barreirinhas, Santo Amaro e Primeira } \\
\text { Cruz }\end{array}$ \\
\hline $\begin{array}{l}\text { Parque Nacional da } \\
\text { Chapada das Mesas }\end{array}$ & $\begin{array}{l}\text { Decreto Federal s/n de } \\
12 / 12 / 2005\end{array}$ & Proteção Integral & Cerrado & 1.600 & Carolina, Riachão e Estreito \\
\hline $\begin{array}{l}\text { Reserva Biológica do } \\
\text { Gurupi }\end{array}$ & $\begin{array}{c}\text { Lei Federal } \mathrm{N}^{\circ} 95.614 \mathrm{de} \\
12 / 01 / 1988\end{array}$ & Proteção Integral & Amazônia & 2.712 & $\begin{array}{c}\text { Bom Jardim, São João do Carú e Centro } \\
\text { Novo }\end{array}$ \\
\hline $\begin{array}{l}\text { Reserva Extrativista do } \\
\text { Quilombo do Frechal }\end{array}$ & $\begin{array}{l}\text { Decreto Federal s/n } 534 \text { de } \\
21 / 05 / 1992\end{array}$ & Uso Sustentável & Amazônia & 9 & Mirinzal \\
\hline $\begin{array}{l}\text { Reserva Extrativista do } \\
\text { Cururupu }\end{array}$ & $\begin{array}{l}\text { Decreto Federal S/N de } \\
\text { 03/06/2004 }\end{array}$ & Uso Sustentável & Amazônia & 1.850 & $\begin{array}{l}\text { Cururupu, Serrano do Maranhão, } \\
\text { Apicum Açu e Bacuri }\end{array}$ \\
\hline $\begin{array}{l}\text { Reserva Extrativista do } \\
\text { Ciriaco }\end{array}$ & $\begin{array}{l}\text { Decreto Federal } \mathrm{N}^{\circ} 534 \text { de } \\
\text { 20/05/1992 alterado por } \\
\text { Decreto S/N 17/06/2010 }\end{array}$ & Uso Sustentável & Amazônia & 8 & Cidelândia \\
\hline $\begin{array}{l}\text { Reserva Extrativista da } \\
\text { Mata Grande }\end{array}$ & $\begin{array}{c}\text { Decreto Federal s/n } 532 \text { de } \\
\text { 20/05/1992 }\end{array}$ & Uso Sustentável & Amazônia & 11 & Senador La Roque e Davinópolis \\
\hline $\begin{array}{l}\text { Reserva Extrativista da } \\
\text { Chapada Limpa }\end{array}$ & $\begin{array}{l}\text { Decreto Federal s/n } 536 \text { de } \\
21 \text { de maio de } 1992\end{array}$ & Uso Sustentável & Cerrado & 120 & Chapadinha \\
\hline
\end{tabular}

Fonte: ICMBIO; SEMA, 2016.

Etapa 2: Nesta etapa foram trabalhados a forma de qualificação e ponderação dos indicadores (Quadro 2). Esse processo ocorreu com base em entrevistas com gestores das 11 UCs em foco, que contaram com a participação da comunidade local e pesquisadores das instituições de pesquisa envolvidas. As entrevistas realizadas tiveram característica informais, sendo estas consideradas do tipo guiada e semidiretivas ${ }^{1}$, com o objetivo de testar, aprofundar e validar as hipóteses do estudo (COHEN et al., 2007).

Etapa 3: Nessa etapa foram empregados testes estatísticos, para sintetização dos indicadores e construção do ICA. No intuito de sintetizar os indicadores, desenvolve-se uma matriz com base na escala de comparação, visando estabelecer linearmente a hierarquia de importância entre os indicadores definidos. Esse procedimento baseou-se em três princípios: decomposição (1), julgamentos comparativos (2) e síntese de prioridades (3), cuja estrutura hierárquica delimita-se em Nível I (Objetivo a ser alcançado), Nível II (critérios) e Nível III (alternativas). Dessa forma, definiu-se a significância de cada variável com sua soma igual a 1 como uma condição necessária para a combinação linear ponderada dos critérios (ARGYRIOU et al., 2016). Após a construção da matriz, foi possível ordenar as alternativas de acordo com seus respectivos níveis de importância.

Para essa fase foram realizados 09 trabalhos de campo com o objetivo de sistematizar entrevistas com gestores e comunidade local, mapear os conflitos e agentes tensores locais, bem como dirimir dúvidas na foto interpretação de imagens de satélite. No processo utilizou-se drone (DJI 4 Phanton Pro) e GPS etrex 30, para posterior construção de banco de dados temático e confecção dos layouts finais dos mapas.

Os dados referentes a dimensão institucional, foram coletados por UC, através de gestores e instituições estaduais e municipais. Em relação as variáveis ligadas a dimensão ambiental, foi avaliado o nível de proteção da área protegida, além de considerar os tipos dos usos existentes nas UCs, sendo estes classificados como vedados ou permitidos de acordo com o Sistema Nacional de Unidades de Conservação

1 Os tópicos e questões tratados foram definidos antecipadamente. O entrevistador decide a sequência das perguntas durante a entrevista, pois é quem conhece os temas sobre os quais tem de obter reações por parte do inquirido, mas a ordem e a forma como serão introduzidos, são escolhidos durante a entrevista (THIOLLENT, 1982). 
(SNUC) e respectivos planos de manejo. Ressalta-se que para construção dos indicadores relativos aos tipos de uso da terra, foram utilizados dados obtidos através do processamento digital das imagens LANDSAT $5 e$ LANDSAT 8 (resolução espacial de 30m). Essas imagens foram selecionadas através do Instituto Nacional de Pesquisas Espaciais - INPE e United States Geological Survey - USGS, em seguida foram processadas através do software Arc Gis 10.6 Isso possibilitou retratar as dimensões, configuração e nível de alteração da paisagem, além de quantificar e mensurar o percentual dos diferentes tamanhos de propriedades por módulos fiscais ${ }^{2}$, existentes nas UCs.

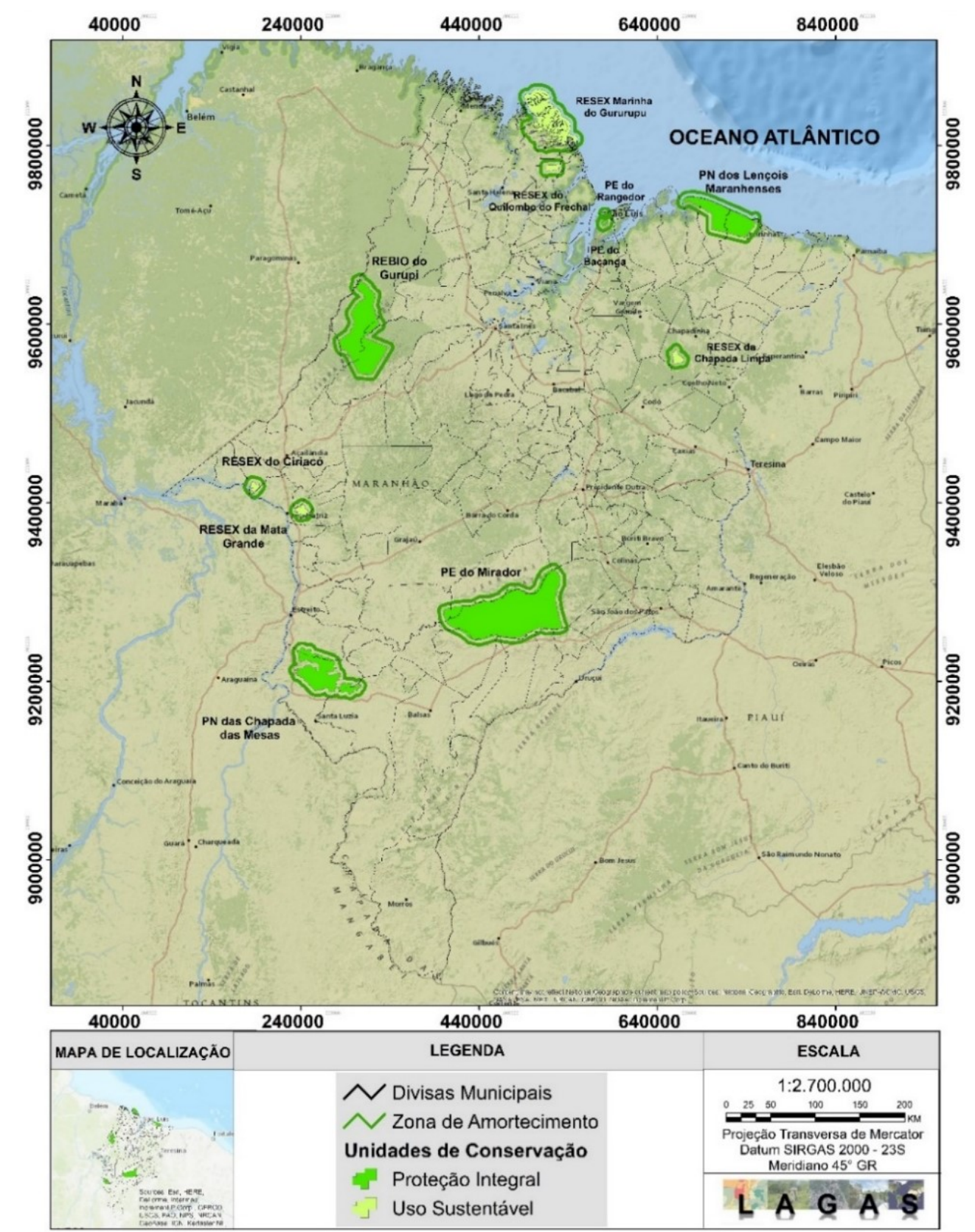

Figura 1: Mapa de Localização das UCs em estudo no Maranhão

Após a qualificação dos indicadores, utilizou-se o método de Analytic Hierarchy Process (AHP) (SAATY, 1991). A utilização do AHP resultou na categorização e ponderação de matrizes de comparação para cada nível, com a definição do grau de importância das variáveis, seguindo a escala de comparadores no Quadro 2 e 3 (SAITO, 2015).

2 Os módulos fiscais seguem a classificação definida pela Lei 8.629, de 25 de fevereiro de 1993. A dimensão dos módulos varia conforme cada município e sua dinâmica rural, cujo tamanho é dado em hectares, considerando-se Minifúndio como o imóvel rural com área inferior a 1 (um) módulo fiscal; Pequena Propriedade, como um imóvel de área compreendida entre 1 (um) e 4 (quatro) módulos fiscais; Média Propriedade como um imóvel rural de área superior a 4 (quatro) e até 15 (quinze) módulos fiscais e Grande Propriedade aqueles imóveis rurais cuja área é superior a 15 (quinze) módulos fiscais. 
Quadro 2: Escala de Comparadores do método AHP.

\begin{tabular}{|c|c|}
\hline VALORES & IMPORTÂNCIA MÚTUA \\
\hline $\mathbf{1 / 9}$ & Extremamente menos importante que \\
\hline $\mathbf{1} / \mathbf{7}$ & Muito fortemente importante que \\
\hline $\mathbf{1 / 3}$ & Fortemente menos importante que \\
\hline $\mathbf{1}$ & Moderadamente menos importante que \\
\hline $\mathbf{3}$ & Igualmente importante a \\
\hline $\mathbf{5}$ & Moderadamente mais importante que \\
\hline $\mathbf{9}$ & Fortemente mais importante que \\
\hline
\end{tabular}

Fonte: Saaty et al. (1991).

Em seguida, para analisar a consistência do método, avaliou-se a probabilidade dos julgamentos terem sido realizados ao acaso. Para tanto, utilizou-se a medida chamada de Razão de Consistência (RC), conforme Saaty e Vargas (2012). Baldioti (2014), expõe uma regra para a avaliação da coerência do julgamento, visando validar o processo, qual seja: $\mathrm{RR} \leq 0,1=$ Julgamento coerente; $0,1<\mathrm{RR}<0,2$ = Julgamento questionável; $R R \geq 0,2=$ Julgamento incoerente. Após a execução do processo acima, alcançou-se o valor de 0,068 da razão de consistência. Desse modo, afirma-se que os pesos calculados para o modelo proposto, tiveram julgamento considerado coerente.

Após a sintetização dos indicadores selecionados, avaliou-se a forma da variação conjunta entre as variáveis e o seu grau de interdependência (Figura 02 e 03). Em seguida, os valores multiplicaram as matrizes de prioridades pelos vetores de atributos das alternativas. Desse modo, para cada alternativa foi obtido a soma ponderada da importância relativa de cada atributo (SOUZA, 2013). Referente aos vetores de prioridades e/ou significância (Tabela 01), estes foram obtidos a partir das matrizes de comparações paritárias, com o autovetor normalizado sendo calculado seguindo a equação abaixo (RIBEIRO, 2017).

$$
\boldsymbol{A} \boldsymbol{w}=\lambda \boldsymbol{M} \boldsymbol{A} \boldsymbol{X}^{\boldsymbol{W}} \square \begin{aligned}
& A \text { é a matriz de comparações paritárias; } \\
& \begin{array}{l}
w \text { é o autovetor principal, referente aos pesos; } \\
\lambda \max \text { é o autovalor principal de } A .
\end{array}
\end{aligned}
$$

Seguindo esse procedimento, os scores foram agregados por camadas, a partir da combinação ponderada e, posteriormente, hierarquizadas e normalizadas conforme a escala de valorização exposta no Quadro 4.

A partir destas etapas, estruturou-se a modelagem dos cálculos expressa abaixo:

$$
\mathbf{I C A}=\frac{1}{n} \sum_{i=1}^{n} X i . F i \quad \begin{aligned}
& \mathrm{Xi}=\text { Variáveis; } \\
& \mathrm{n}=\text { Numero de indicadores; } \\
& \mathrm{Fi}=\text { Significância dos indicadores. }
\end{aligned}
$$

A metodologia utiliza a escala de valorização adaptada por Padovan (2001), ou seja, criou-se a hierarquização das variáveis a partir do desenvolvimento de cenários, respeitando a categoria, o nível de proteção e as características territoriais (Quadro 5). 
Quadro 3: Qualificação das variáveis selecionadas.

\begin{tabular}{|c|c|c|c|c|}
\hline $\begin{array}{l}\text { DIMENSÃO } \\
\text { AMBIENTAL }\end{array}$ & \multicolumn{2}{|c|}{ QUALIFICAÇÃO } & VALORAÇÃO & JUSTIFICATIVA \\
\hline \multirow[b]{3}{*}{ Área ocupada } & \multicolumn{2}{|c|}{ Não existe / População Tradicional } & 1 & \multirow{12}{*}{$\begin{array}{l}\text { Os tipos dos usos existentes nas UCs } \\
\text { provocam significativos alterações a } \\
\text { dinâmica da paisagem. Os diferentes } \\
\text { tipos de usos foram considerados } \\
\text { vedados ou permitidos, de acordo com a } \\
\text { legislação vigente. }\end{array}$} \\
\hline & \multicolumn{2}{|c|}{ Ocupações irregulares } & 2 & \\
\hline & \multicolumn{2}{|c|}{$\begin{array}{l}\text { Grandes empreendimentos imobiliários } \\
\text { (condomínios), rurais (fazendas) e industriais na área } \\
\text { intra-UC e entorno }\end{array}$} & 3 & \\
\hline \multirow{3}{*}{ Pecuária } & \multicolumn{2}{|c|}{ Não existe / pequena propriedade } & 1 & \\
\hline & \multicolumn{2}{|c|}{ Média propriedade } & 2 & \\
\hline & \multicolumn{2}{|c|}{ Grande propriedade } & 3 & \\
\hline \multirow{3}{*}{ Agricultura } & \multicolumn{2}{|c|}{ Não existe / pequena propriedade } & 1 & \\
\hline & \multicolumn{2}{|c|}{ Média propriedade } & 2 & \\
\hline & \multicolumn{2}{|c|}{ Grande propriedade } & 3 & \\
\hline \multirow{3}{*}{ Extrativismo } & \multicolumn{2}{|c|}{ Não existe / pequena propriedade } & 1 & \\
\hline & \multicolumn{2}{|c|}{ Média propriedade } & 2 & \\
\hline & \multicolumn{2}{|c|}{ Grande propriedade } & 3 & \\
\hline \multirow{4}{*}{$\begin{array}{l}\text { Área com floresta/ } \\
\text { natural não vegetado }\end{array}$} & Proteção Integral & Uso Sustentável & & \multirow{4}{*}{$\begin{array}{l}\text { Refere-se a áreas com padrão de } \\
\text { cobertura da terra compatíveis com } \\
\text { diferentes fitofisionomias, ainda que } \\
\text { apresentem algum nível de alteração } \\
\text { produtiva pouco intensiva ou de } \\
\text { subsistência, como pastagens naturais, } \\
\text { áreas não vegetadas como praias } \\
\text { fluviais, afloramentos rochosos e dunas, } \\
\text { demonstrando o grau de transformação } \\
\text { da paisagem ocasionado pelas pressões } \\
\text { socioculturais. }\end{array}$} \\
\hline & Satisfatório (>80\%) & Satisfatório (>35\%) & 1 & \\
\hline & $\begin{array}{l}\text { Medianamente satisfatório } \\
\qquad(40 \% \text { A } 79 \%)\end{array}$ & $\begin{array}{l}\text { Medianamente } \\
\text { satisfatório }(21 \% \mathrm{~A} \\
34 \%)\end{array}$ & 2 & \\
\hline & $\begin{array}{l}\text { Pouco satisfatório }(20 \% \mathrm{~A} \\
39 \%)\end{array}$ & $\begin{array}{l}\text { Pouco satisfatório } \\
\qquad(11 \% \text { A } 20 \%)\end{array}$ & 3 & \\
\hline \multirow{3}{*}{$\begin{array}{l}\text { Área prioritárias para } \\
\text { Conservação }\end{array}$} & \multicolumn{2}{|c|}{ Extremamente alta prioridade } & 1 & \multirow{3}{*}{$\begin{array}{c}\text { Indica o nível de representatividade das } \\
\text { unidades de conservação, considerando } \\
\text { objetivos de conservação, eficiência, } \\
\text { metas, grau de insubstituibilidade e } \\
\text { vulnerabilidade. }\end{array}$} \\
\hline & \multirow{2}{*}{\multicolumn{2}{|c|}{ Não se localiza em áreas prioritária para conservação }} & 2 & \\
\hline & & & 3 & \\
\hline $\begin{array}{c}\text { DIMENSÃO } \\
\text { POLÍTICA E } \\
\text { INSTITUCIONAL }\end{array}$ & QUALIFIC & $\tilde{\mathbf{A}} \mathbf{O}$ & VALORAÇÃO & JUSTIFICATIVA \\
\hline & 5 Ano & & 1 & $\begin{array}{l}\text { De acordo com a } \operatorname{art}^{\circ} 2 \text { da Lei } N^{\circ} 9.985 \\
\text { (SNUC) o plano de manejo é o }\end{array}$ \\
\hline Plano de Manejo & Acima de 5 & & 2 & $\begin{array}{l}\text { documento técnico que fundamenta os } \\
\text { objetivos gerais da UC, estabelecendo o } \\
\text { zoneamento, as normas o uso da área e o }\end{array}$ \\
\hline & Não pos & & 3 & $\begin{array}{c}\text { manejo dos recursos naturais, a } \\
\text { implantação das estruturas físicas } \\
\text { necessárias ao monitoramento e gestão } \\
\text { da unidade. }\end{array}$ \\
\hline & $\begin{array}{l}\text { Existe e desenvolve ações } \\
\text { junto à comuni }\end{array}$ & $\begin{array}{l}\text { gestão do território } \\
\text { le local }\end{array}$ & 1 & $\begin{array}{l}\text { A existência e manutenção do conselho } \\
\text { gestor garante a participação no } \\
\text { planejamento e na gestão de }\end{array}$ \\
\hline Conselho Gestor & $\begin{array}{l}\text { Existe e não desenvolve ne } \\
\text { gestão do te }\end{array}$ & $\begin{array}{l}\text { Im tipo de ações de } \\
\text { ório }\end{array}$ & 2 & $\begin{array}{l}\text { representantes de órgãos públicos e da } \\
\text { sociedade civil, ampliando o } \\
\text { monitoramento e fiscalizacão das UCs }\end{array}$ \\
\hline & Não exi & & 3 & em escala local e regional. \\
\hline Secretaria de Meio & $\begin{array}{l}\text { Sim existe (Desenvolve } \\
\text { monitoramento e }\end{array}$ & $\begin{array}{l}\text { jetos e atua no } \\
\text { calização) }\end{array}$ & 1 & $\begin{array}{l}\text { A existência Secretaria Municipal de } \\
\text { Meio Ambiente é um instrumento de } \\
\text { ordenamento territorial na área de }\end{array}$ \\
\hline Ambiente & Estrutura em Conjunto com & tra Secretaria (SIM) & 2 & $\begin{array}{l}\text { entorno da UC, possibilitando o } \\
\text { monitoramento e a fiscalizacão das UCs }\end{array}$ \\
\hline & Não exi & & 3 & em nível municipal. \\
\hline & Executa & & 1 & \\
\hline Situação Fundiária & Parcialmente & cutada & 2 & Indica se a situaçáo fundiaria da UC esta \\
\hline & Não execl & & 3 & \\
\hline & Satisfa & & 1 & \\
\hline Desenho da UC & Satisfaz parc & nente & 2 & $\begin{array}{l}\text { Indica se a localização e os limites da } \\
\text { UC são coerentes com os seus objetivos }\end{array}$ \\
\hline & Não sati & & 3 & \\
\hline & Satisfa & & 1 & Indica se a estrutura da UC possibilita a \\
\hline Recursos Humanos e & Satisfaz parc & nente & 2 & realização de ações de manejo \\
\hline Infraestrutura & Não sati & & 3 & $\begin{array}{l}\text { adequados a categoria e demandas } \\
\text { existentes na UC. }\end{array}$ \\
\hline & Satisfa & & 1 & Indica se ações de manejo estão de \\
\hline Ações de manejo & Satisfaz parc & nente & 2 & acordo com o Plano de Manejo e as \\
\hline & Não sati & & 3 & demandas existentes na UC. \\
\hline Capacidade de & Satisfa & & 1 & Indica se a capacidade de \\
\hline monitoramento e & Satisfaz parc & nente & 2 & monitoramento e fiscalização atende as \\
\hline fiscalização & Não sati & & 3 & \\
\hline
\end{tabular}




\begin{tabular}{|c|c|c|c|c|c|c|c|c|c|c|c|c|c|c|}
\hline INDICADORES & 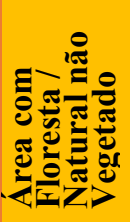 & 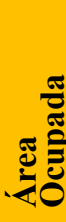 & لَّ & 焉 & : & 离 & 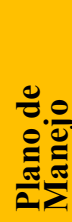 & 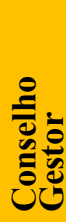 & & & 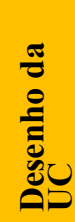 & 竞 & 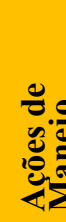 & 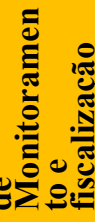 \\
\hline $\begin{array}{l}\text { Área com floresta/ } \\
\text { natural não vegetado }\end{array}$ & 1 & $1 / 3$ & $1 / 3$ & $1 / 3$ & 3 & $1 / 9$ & $1 / 5$ & $1 / 5$ & 3 & $1 / 3$ & $1 / 9$ & $1 / 3$ & $1 / 5$ & $1 / 5$ \\
\hline Área ocupada & 3 & 1 & 1 & 3 & 3 & $1 / 3$ & 3 & $1 / 3$ & 5 & $1 / 3$ & $1 / 3$ & 3 & 3 & 5 \\
\hline Pecuária & 3 & $1 / 3$ & 1 & $1 / 3$ & 3 & $1 / 3$ & 3 & $1 / 3$ & 5 & $1 / 5$ & $1 / 3$ & 3 & 3 & 5 \\
\hline Agricultura & $1 / 3$ & $1 / 3$ & $1 / 3$ & 1 & 3 & $1 / 3$ & 3 & $1 / 3$ & 5 & $1 / 5$ & $1 / 3$ & 3 & 3 & 5 \\
\hline Extrativismo & $1 / 3$ & $1 / 3$ & $1 / 3$ & $1 / 3$ & 1 & $1 / 3$ & 3 & $1 / 3$ & 5 & $1 / 5$ & $1 / 3$ & 3 & 3 & 5 \\
\hline $\begin{array}{l}\text { Área prioritária para } \\
\text { Conservação }\end{array}$ & 9 & $1 / 5$ & $1 / 5$ & $1 / 5$ & $1 / 5$ & 1 & $1 / 3$ & 3 & 9 & 3 & 1 & 5 & 5 & 9 \\
\hline Plano de Manejo & 5 & $1 / 3$ & $1 / 3$ & $1 / 3$ & $1 / 3$ & $1 / 5$ & 1 & $1 / 3$ & 3 & $1 / 3$ & $1 / 3$ & $1 / 3$ & $1 / 3$ & 3 \\
\hline Conselho Gestor & 5 & $1 / 3$ & $1 / 3$ & $1 / 3$ & $1 / 3$ & $1 / 5$ & 1 & 1 & 5 & $1 / 3$ & $1 / 3$ & 3 & 3 & 3 \\
\hline SEMAM & $1 / 3$ & $1 / 5$ & $1 / 5$ & $1 / 5$ & $1 / 5$ & $1 / 9$ & $1 / 3$ & 5 & 1 & $1 / 5$ & $1 / 5$ & $1 / 3$ & $1 / 3$ & $1 / 3$ \\
\hline Situação Fundiária & 3 & 1 & 1 & 1 & 1 & $1 / 5$ & 3 & 3 & 3 & 1 & 1 & 5 & 3 & 3 \\
\hline Desenho da UC & 9 & $1 / 5$ & $1 / 5$ & $1 / 5$ & $1 / 5$ & 1 & $1 / 5$ & 3 & 9 & 5 & 1 & 5 & 3 & 5 \\
\hline RH e Infraestrutura & 3 & 1 & 1 & 1 & 1 & $1 / 3$ & $1 / 3$ & $1 / 3$ & 5 & $1 / 5$ & $1 / 5$ & 1 & $1 / 5$ & 1 \\
\hline Ações de manejo & 5 & $1 / 3$ & $1 / 3$ & $1 / 3$ & $1 / 3$ & $1 / 3$ & $1 / 3$ & $1 / 3$ & 3 & $1 / 3$ & $1 / 3$ & 3 & 1 & 3 \\
\hline $\begin{array}{l}\text { Capacidade de } \\
\text { monitoramento e } \\
\text { fiscalização }\end{array}$ & 5 & $1 / 3$ & $1 / 3$ & $1 / 3$ & $1 / 3$ & $1 / 3$ & $1 / 3$ & 1 & 3 & $1 / 3$ & $1 / 3$ & 1 & $1 / 3$ & 1 \\
\hline
\end{tabular}

Figura 2: Matriz de Variáveis.

DIMENSÃO

INDICADOR

\section{UCs}

Resex do Ciricó

Resex da Mata Grande

Resex Quilombo do

Frechal

Resex do Cururupu

Reserva Biológica do

Gurupi

Parque Estadual do

Bacanga

PE do Rangedor

PARNA Lençóis

Maranhenses

PE do Mirador

Resex da Chapada Limpa PARNA da Chapada das Mesas

\section{Dimensão Ambiental}

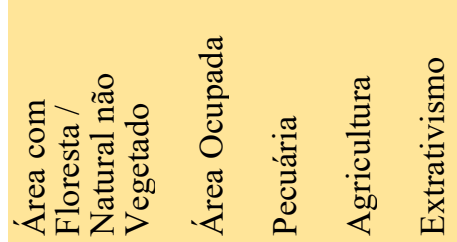

\section{Dimensão Institucional}

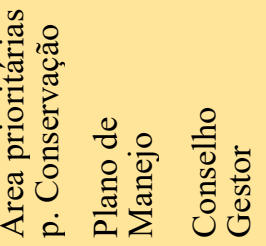

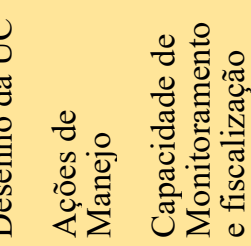


Tabela 1: Nível de significância das variáveis do ICA

\begin{tabular}{|c|c|c|c|c|c|}
\hline DIMENSÃO & INDICADORES & $\begin{array}{l}\text { Média } \\
\text { geométrica de } \\
\text { cada linha }\end{array}$ & $\begin{array}{c}\text { Autovetor de } \\
\text { cada linha } \\
\text { dividido pelo } \\
\text { total }\end{array}$ & $\begin{array}{l}\text { Soma de cada } \\
\text { coluna da } \\
\text { matriz }\end{array}$ & $\begin{array}{l}\text { Autovetor da linha } \\
\text { multiplicado pela } \\
\text { soma de cada coluna }\end{array}$ \\
\hline \multirow{6}{*}{ Ambiental } & Área Ocupada & 1,409 & 0,071 & 13 & 0,925 \\
\hline & $\begin{array}{c}\text { Área Natural / Natural não } \\
\text { Vegetados }\end{array}$ & 0,463 & 0,023 & 67 & 1,566 \\
\hline & $\begin{array}{l}\text { Área Prioritária para } \\
\text { Conservação }\end{array}$ & 1,736 & 0,088 & 7,1 & 0,621 \\
\hline & Pecuária & 1,153 & 0,058 & 13,7 & 0,796 \\
\hline & Agricultura & 1,027 & 0,052 & 15,7 & 0,813 \\
\hline & Extrativismo & 0,915 & 0,046 & 23,7 & 1,094 \\
\hline \multirow{8}{*}{ Institucional } & Plano de Manejo & 0,726 & 0,037 & 30,6 & 1,122 \\
\hline & Conselho Gestor & 0,996 & 0,050 & 20,4 & 1,025 \\
\hline & $\begin{array}{c}\text { Secretaria Municipal de Meio } \\
\text { Ambiente }\end{array}$ & 0,293 & 0,015 & 93 & 1,374 \\
\hline & Desenho da UC & 1,683 & 0,085 & 8,7 & 0,739 \\
\hline & Recursos Humanos & 0,691 & 0,035 & 47 & 1,640 \\
\hline & Situação Fundiária & 2,001 & 0,101 & 13,9 & 1,409 \\
\hline & Ações de Manejo & 0,860 & 0,043 & 45,9 & 1,996 \\
\hline & Capacidade de Monitoramento & 0,630 & 0,032 & 70,2 & 2,233 \\
\hline
\end{tabular}

Quadro 4: Escala de valorização utilizada para mensurar o nível do ICA.

\begin{tabular}{|c|c|c|}
\hline Nível & Variação (0 A 1) & ICA \\
\hline $\mathbf{1}$ & $>0,800$ & Satisfatório \\
\hline $\mathbf{2}$ & $0,551 \mathrm{~A} 0,800$ & Medianamente satisfatório \\
\hline $\mathbf{3}$ & $0,401 \mathrm{~A} 0,551$ & Pouco satisfatório \\
\hline $\mathbf{4}$ & $<0,400$ & Insatisfatório \\
\hline
\end{tabular}

Fonte: Adaptado PADOVAN, 2001.

Quadro 5: Descrição do nível de efetividade de acordo com a escala de valorização.

\section{Nível 1: Satisfatório $(>0,800)$}

Indica que a UC conta com os requerimentos institucionais necessários para estabelecer um manejo eficiente, cumprindo com os objetivos da UC, com a execução de políticas públicas, ações de gestão e manejo que atendam as demandas da sociedade. Garante a conservação dos seus recursos naturais, além de demonstrar a existência de diferentes tipos de usos que estão alinhados com os objetivos da categoria e da UC.

\section{Nível 2: Medianamente Satisfatório $(0,551$ A 0,800$)$}

Indica que a UC conta com os requerimentos institucionais necessários desatualizados impossibilitando o estabelecimento de um manejo adequado. Contudo, os objetivos de criação da UC se encontram em patamares mínimos para a sua conservação, onde o modelo de gestão instituído cumpre com os objetivos da UC e garante a conservação dos recursos naturais, além de demonstrar a existência de usos que apesar de serem vedados conforme a categoria da UC, não impedem o alcance dos objetivos da UC.

\section{Nível 3: Pouco Satisfatório $(0,401$ A 0,550$)$}

Indica que a UC não conta com os requerimentos institucionais mínimos necessários para estabelecer um manejo adequado. Contudo, o modelo de gestão instituído cumpre com os objetivos da UC e garante parcialmente a conservação dos recursos naturais, além de demonstrar a existência de diferentes usos vedados conforme a categoria da UC, os quais dificultam o alcance dos objetivos da área protegida.

\section{Nível 4: Insatisfatório $(<0,400)$}

Indica que a UC se encontrar em situação de dificuldade na gestão dos seus objetivos e apresenta um baixo desempenho de retorno da política pública para a sociedade. A UC não contar com os requerimentos institucionais mínimos necessários para estabelecer um manejo adequado, além de demonstrar a existência de diferentes usos vedados conforme a categoria da UC, os quais resultam em alto nível de alteração da paisagem, impedindo o alcance dos objetivos da área protegida.

Fonte: Adaptado PADOVAN, 2001; ICMBIO/WWF, 2017

Os procedimentos aplicados permitem a aplicação da metodologia em escala regional, servindo como base de referência, comparação e nível de associação espacial. Para tanto, utilizou-se a técnica de análise geoestatística, conhecida como Krigagem, através da ferramenta Geostatistic Analyst do ArcGIS 10.6, com o objetivo de regionalizar as UCs com base no ICA, conforme Yamamoto e Landim (2013). 


\section{Resultados e Discussões}

Neste tópico o que se propõe é um modo de apresentar simultaneamente o índice sintético e os indicadores por dimensão (ambiental e institucional), de modo que seja possível analisar os diferentes tipos de uso e a capacidade de governança das UCs do Maranhão, como podemos analisar no Gráfico 1.

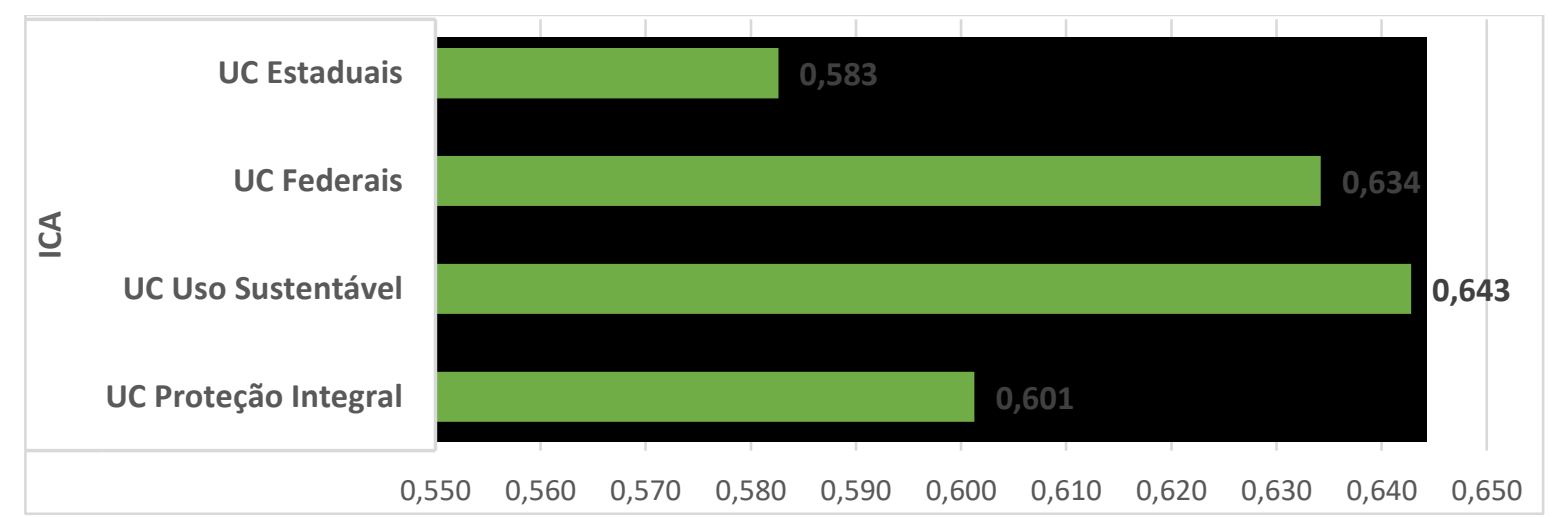

Gráfico 1: ICA por tipo governança e nível de proteção das UCs do Maranhão.

No que tange à instância política responsável pela gestão das UCs no Maranhão, vinculadas diretamente à secretaria de estado apresentaram índice medianamente satisfatório, porém muito próximo do patamar abaixo (pouco satisfatório), isso por não apresentarem os elementos principais de garantias de um melhor convívio entre o uso social e a proteção da biodiversidade, como planos de manejo, conselhos gestores, participação social local e planejamento participativo. Isso vem de encontro à modelagem proposta por Oakerson (1992) que vem recebendo contribuições (OSTROM e COX, 2010; GRAEME et al., 2015) que considera as variáveis interdependentes: atributos físicos e tecnológicos, tomadas de decisão via institucionalização de gestão, interação dos atores sociais envolvidos e resultados todos têm precária efetividade quando de responsabilidade estadual.

Observa-se que as UCs de Uso Sustentável apresentaram ICA de 0,643, maior que as de Proteção Integral, com 0,601. Este resultado demonstra que as formas de ocupação diferenciada das UCs de uso sustentável possibilitaram maior capacidade de conservação do território. Os indicadores que contribuíram favoravelmente foram o alinhamento dos diferentes tipos de uso da terra, a existência dos instrumentos de planejamento e gestão, além da maior contribuição da comunidade no conselho gestor e na mediação de conflitos para otimizar as ações de manejo.

Os dados obtidos demonstram boas evidências de que esses territórios alcançaram significativos níveis de conservação da biodiversidade, corroborando com os resultados obtidos por Geldmam et al. (2017), no que tange aos resultados das APs em nível mundial. Contribuindo com essa perspectiva Stoll-Kleemann e Job (2008), demonstram que a efetividade da proteção da biodiversidade, por meio das áreas protegidas, está intrisecamente relacionada a gestão local empática, que é influenciada pela melhora dos meios de subsistência locais, possibilitado pelo modelo de uso sustentável instituído.

Ressalta-se que existe um consenso, sobre a relevância do envolvimento das comunidades locais na gestão e no desenvolvimento de ações, ligadas ao monitoramento e fiscalização das APs, como demonstram Diegues (2000); Mcnelly (2004); Sherl et al. (2006); Marinelli (2011). No entanto, pelo grande número de UCs de proteção integral e pela ainda pouca participação da comunidade, Bertzky et al. (2015) observa que em geral isso não ocorre. Esse cenário acaba por perpetuar os elevados percentuais de pobreza e incidência de conflitos, identificados pelo baixo patamar dentro do nível medianamente satisfatório alcançados.

Esses resultados demonstraram índices distintos nas dimensões institucional e ambiental, quando na escala da produção e reprodução da dinâmica territorial que caracterizam as regiões onde as APs estão inseridas (GRAEME et al., 2015; GORDON et al., 2018). Sob esse entendimento, buscou-se regionalizar as UCs a partir do ICA, com base no cálculo do desvio padrão entre os diferentes níveis de efetividade das UCs, localização, distância e a semivariância (Figura 4).

A regionalização das UCs por ICA, apresenta não só como as UCs estão distribuídas no território do estado, mas demonstra como as diferentes dinâmicas territoriais influenciam no alcance dos objetivos das destas áreas protegidas. Observa-se que ao norte encontram-se as UCs que contam com os requerimentos institucionais necessários para estabelecer um manejo eficiente, cumprindo com os objetivos da UC, com a execução de políticas públicas, ações de gestão e manejo. Elas são influenciadas principalmente pelo maior 
monitoramento e fiscalização, além de contar com a existência de diferentes tipos de usos que estão alinhados com os objetivos da categoria e da UC. Na porção centro-sul e oeste do estado, elas caracterizamse por não contarem com os requerimentos institucionais, necessários para estabelecer um manejo adequado e reduzida capacidade de fiscalização, o que possibilita os avanços de diferentes usos da terra vedados conforme a categoria da UC, deflagrando significativas alterações da paisagem local e impossibilitando o alcance dos objetivos desta (Gráfico 2).

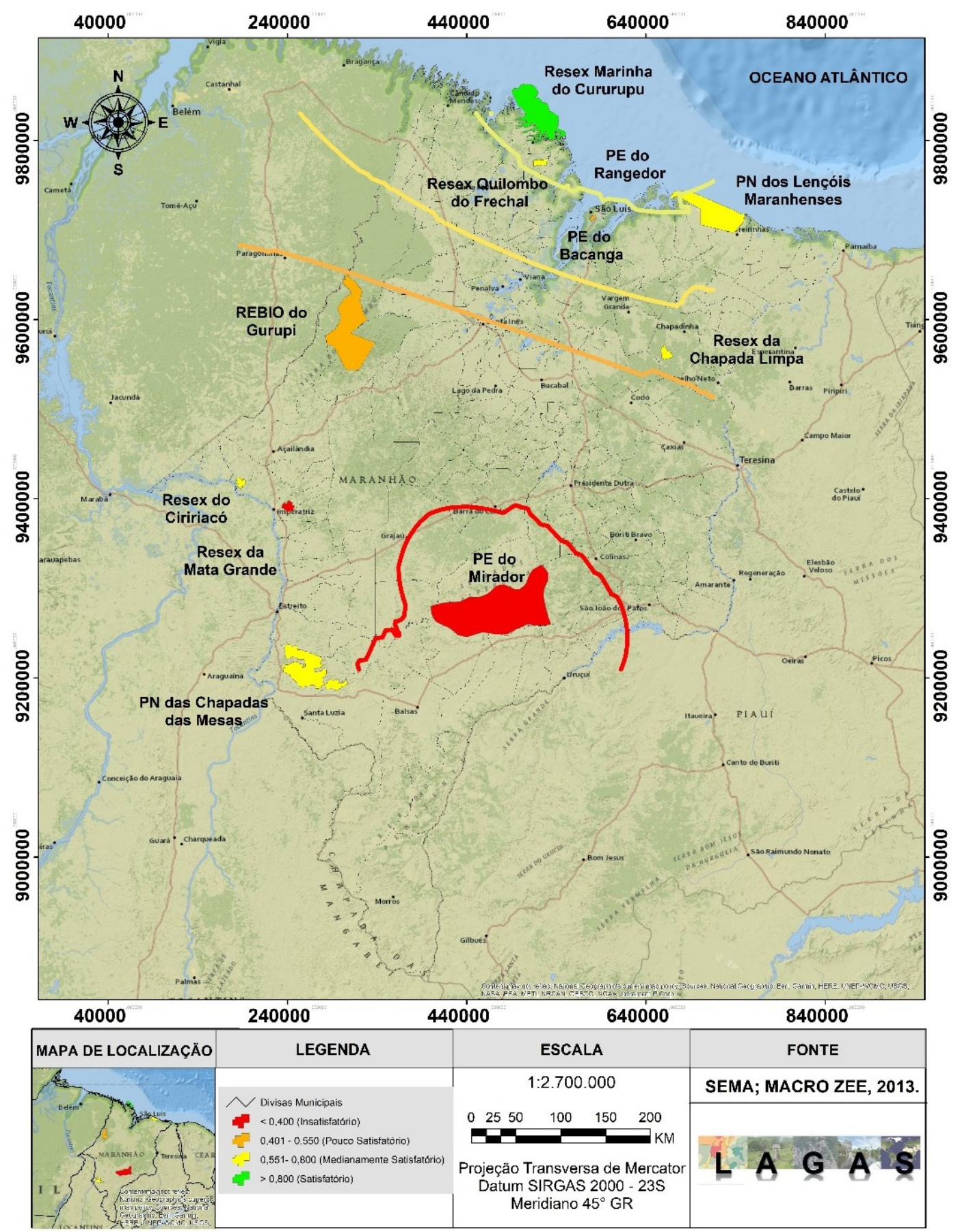

Figura 4: Regionalização das Unidades de Conservação do Maranhão, com base no ICA.

O PE do Rangedor $(0,968)$ é a UC do Maranhão com maior nível de ICA. Está UC localiza-se na área urbana de São Luís (capital maranhense), próximo a faixa de praias cercado por bairros de classe média-alta, onde mesmo pressionada pela especulação imobiliária em busca de novos espaços residenciais e crescente pressão de diversos agentes, é a única unidade de proteção integral do Maranhão que não registra invasões. Entretanto essa área protegida tornou-se uma verdadeira "ilha", assim como revela estudo desenvolvido pelo WWF (2000), no qual afirma que cerca de $41 \%$ dessa categoria de UCs têm mais da metade da área de seu entorno desmatada. 
Entre as unidades de Uso Sustentável, destaca-se a Resex do Cururupu $(0,856)$, com nível alto de efetividade satisfatório. Essa Resex, composta por um arquipélago de ilhas de sedimentação dunar quaternária, que interligam um vasto manguezal das reentrâncias maranhenses, apresenta contenção do desmatamento na região, conselho gestor participativo, plano de manejo em vias de se consolidar e construído coletivamente. A base da subsistência é a pesca e, em certa medida, a silvicultura e extrativismo vegetal.

No litoral oriental do Maranhão, o PARNA dos Lençóis Maranhenses $(0,748)$ apresentou ICA medianamente satisfatório. Esse resultado é influenciado pelo significativo controle no avanço da ocupação na área protegida a despeito do aumento das pressões na área de entorno, por parte da especulação imobiliária e instalação de grandes projetos estaduais e privados, em parte esse cenário justifica-se pelos impositivos aspectos físicos (ambiente dunário) da região. Contudo, identifica-se plano de manejo desatualizado, reduzida ação do conselho gestor e infraestrutura insuficiente para fiscalização.

Já na região sudoeste do Maranhão onde se encontra a Resex do Ciriacó $(0,737)$ e Mata Grande $(0,313)$, há grande influência da instalação de empresas siderúrgicas atraídas pela cadeia produtiva que gravita no entorno da estrada de Ferro Carajás e a Suzano Papel e Celulose, bem como do polo gesseiro e comercial. A região vem passando por diversas obras de infraestrutura viária, como a estrada do Arroz e o anel da soja. Enquanto que as UCs da região oeste (Amazônia maranhense) e centro-sul do estado apresentam alto nível de alteração da paisagem, impedindo o alcance dos objetivos da área protegida. Entre elas estão a REBIO do Gurupi $(0,451)$, cujo o nível do ICA se vincula à insuficiente fiscalização e deficiências nos instrumentos institucionais de gestão e/ou participativo, já o PE do Mirador $(0,275)$, apresenta maior influência do indicador desenho da UC (limites e localização) não possibilita a proteção de grande parte dos mananciais do rio Itapecuru (principal objetivo), bem como ausência dos instrumentos de planejamento e gestão necessários para fiscalização, como plano de manejo e conselho gestor. Esse cenário solidifica as bases para a produção e reprodução de conflitos fundiários e sociais, que produzem significativas ameaças socioeconômicas e ambientais.

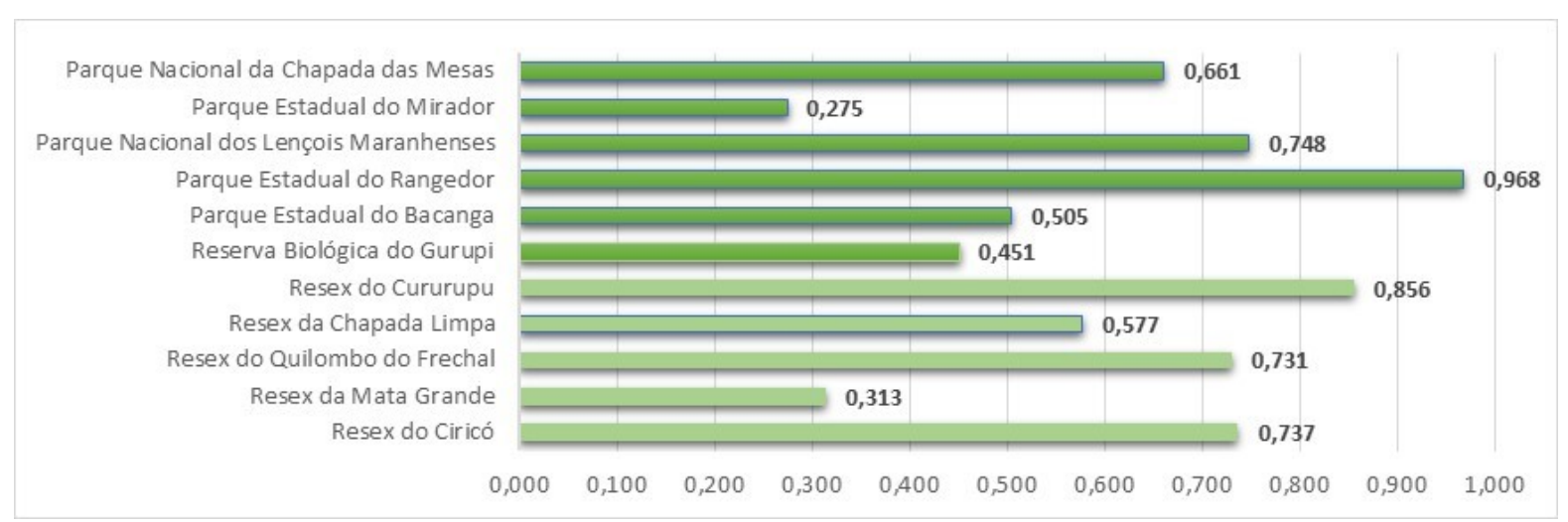

Gráfico 2: Comparativo do ICA entre as Unidades de Conservação em análise

\section{Conclusões}

A construção do ICA aplicado ao Maranhão representa uma nova possibilidade de ponderar quantitativamente variáveis mediante correlações qualitativas, organizando e estabelecendo um modelo relacional de combinação de dados. Considerando as escalas de aplicação de diferentes técnicas, os resultados alcançados na presente pesquisa, demonstraram nível satisfatório, apresentando conclusões similares aos obtidos por metodologias já consolidadas e aplicadas no Brasil e no mundo (WWF, 1999; RAPPAM, 2005-2010-2015 e SAMGe, 2017). Contudo, ressalta-se que a metodologia proposta apresentou entre outras vantagens, a redução da subjetividade no processo de avaliação da efetividade, aumento da confiabilidade e a capacidade de reprodução, além de rápida aplicação.

Entretanto, a metodologia também apresentou limitações que devem ser consideradas. Entre as lacunas identificadas no conjunto de dados coletados não foi possível ser mais específico, em relação a capacidade e as necessidades de infraestrutura de recursos humanos das UCs. Isso se deu por ser um dado que, além de variar bastante no decorrer do ano, não é fornecido e atualizado periodicamente pelos gestores. Dessa forma, não foi possível, ainda, identificar uma base de dados secundárias, originária de fonte confiável, para reprodução e tratamento das variáveis, para o ano base utilizado na construção do ICA. 
Ultimando, no contexto geral, o desenvolvimento do ICA demonstrou que a avaliação dos tipos de usos e a estrutura de governança da UC deve ser compreendida em escala local e regional, considerando processos endógeno e as singularidades territoriais. Desse modo, a proposta metodológica demonstrou formas de atenuar a subjetividade das interpretações, através do estabelecimento de uma hierarquia de soluções com a quantificação dos atributos e suas correlações, buscando assim, contribuir conceitualmente e metodologicamente com o desenvolvimento de políticas públicas, a partir das Unidades de Conservação e não apesar delas.

\section{Agradecimentos}

Ao apoio da Coordenação de Aperfeiçoamento de Pessoal de Nível Superior - Brasil (CAPES) - Código de Financiamento 001, ao LAGAS/UnB e projetos UnB/IRD JEAI-GITES / LMI-Sentinela.

\section{REFERÊNCIAS}

ARGYRIOU, A.V. et al. GIS multicriteria decision analysis for evaluation and mapping of landscape deformation neotectonics: A case study from Crete. Geomorphology, p. 262 -274, 2016.

BALDIOTI, H.R. 2014. Abordagem Multicritério para Avaliação de Modelos Geradores de Cenários Aplicados ao Planejamento da Operação Hidrotérmica de Médio Prazo. Dissertação (Mestrado) Pontifícia Universidade Católica do Rio de Janeiro, Rio de Janeiro.

BERTZKY, B. et al. Protected Planet Report 2012: Tracking progress towards global targets for protected areas. Switzerland: Cambridge, 2012, 68p.

COHEN, L. et al Research methods in education. Londres: Routledge, 2007, 656p.

DIEGUES, A. C. S. O mito moderno da natureza intocada. Núcleo de Apoio à Pesquisa sobre Populações Humanas e Áreas Úmidas Brasileiras. São Paulo: Hucitec, 2000, 161p.

ERVIN, J. WWF Rapid assessment and priorization of protected are management (Rappam) methodology. Swizertland: Gland, WWF, 2003, 70p.

GELDMANN, J. et al. A global analysis of management capacity and ecological outcomes in terrestrial protected areas. Conservation Letters, p. 1 - 10, 2017.

GORDON, J. E. et al. Geoheritage Conservation and Environmental Policies: Retrospect and Prospect. In: REYNARD E.; BRILHA, J. Editors, Geoheritage. Chennai: Elsevier, p. 213-236, 2018.

GRAEME, S. et al. Understanding protected area resilience: a multi-scale, social-ecological approach. Ecological Applications, v. 25, n.2, p. 299-319, 2015.

HOCKING, M. et al. Evaluating Effectiveness: A framework for assessing management effectiveness of protected areas. Suíça: IUCN - Gland, 2006.

ICMBio - Instituto Chico Mendes de Conservação da Biodiversidade. Avaliação comparada das aplicações do método Rappam nas unidades de conservação federais, nos ciclos 2005-06 e 2010. Brasília: ICMBio, 2011, 134p.

LEVERINGTON, F. et al. A Global Analysis of Protected Area Management Effectiveness. Environmental Management, 2010.

LEMOS DE SÁ, R. (2000). Unidades de conservação: espaços ameaçados ou áreas protegidas. (Relatório Técnico). Brasília: 32 p.

MACKINNON, J. et al. Manejo de Áreas Protegidas En Los Trópicos. Suisse: International Union For Conservation Of Nature, 1990, 314p.

MARINELLI, C. E. De olho nas unidades de conservação: Sistema de Indicadores Socioambientais para Unidades de Conservação da Amazônia Brasileira. São Paulo: Instituto Socioambiental, 2011, $12 \mathrm{p}$.

MCNEELY, J. A. (Org.). At least do no harm: poverty and protected areas in China. Discussion paper for the CCICED. Protected Areas Task Force. 20004 
MORIN, E. et al. Educar na era planetária: o pensamento complexo como método de aprendizagem pelo erro e incerteza humana. Trad. Sandra Trabucco Mayra Valenzuela. São Paulo: Cortez;, 2003, $111 \mathrm{p}$.

OAKERSON, R. J. Analyzing the commons. A framework. In: Bromley, D. A. et al., Making the commons work: theory, practice and policy. San Francisco: ICS Press. 1992.

OSTROM, E. et. al. (Eds.) The drama of the commons. Washington: National Research Council, 2001.

OSTROM, E.; COX, M. Moving beyond panaceas: a multi-tiered diagnostic approach for social-ecological analysis. Environmental Conservation, n. 37, p. 451-463, 2010.

PADOVAN, M. P. Formulacion de um estandar y um procedimento para la certificacion del manejo de áreas protegidas. Costa Rica: Centro Agronomico Tropical de Investigacion y Ensenanza, 2001.

RIBEIRO, B. A. Proposta para revelar as preferências de comitês de especialistas a partir do método AHP: uma aplicação ao setor elétrico. Tese (doutorado). Pontifícia Universidade Católica do Rio de Janeiro. Departamento de Engenharia Elétrica. Rio de Janeiro. 2017. 115p.

SAATY, T. L. Método de Análise Hierárquica. 1ª ed. São Paulo: McGraw-Hill, 1991

SAATy, T.L. \& VARGAS, L. G., Models, Methods, Concepts \& Applications of the Analytic Hierarchy Process. $2^{\mathrm{a}}$ ed. New York: Springer. 2012.

SAHOO. S. et al. Environmental vulnerability assessment using Grey Analytic Hierarchy Process based model. Environmental Impact Assessment Review, p. 145-154, 2016.

SCHULZE, K. et al. Na assessment of threats to terrestrial protected áreas. Conservation Letters, p. 1-10, 2017.

SCHAEFER, M. et al. Nature as capital: advancing and incorporating ecosystem services in United States federal policies and programs. PNAS. p. 7383-7389, 2015.

SCHERL, L. M. et al. As áreas protegidas podem contribuir para a redução da pobreza? Oportunidades e limitações. Suíça e Cambridge: IUCN, 2006, 60p.

SOUZA, C.M.P. d., Modelo de Previsão de Despacho de Usinas Termelétricas por meio do Método Multicritério ANP. Dissertação de Mestrado, IBMEC, Rio de Janeiro. 2013.

STOLL-KLEEMANN, S. Evaluation of management effectiveness in protected areas: Methodologies and results. Basic and Applied Ecology, n. 11, p. 377-382, 2010.

Stolton, S. et al. Reporting Progress in Protected Areas a Site Level Management Effectiveness Tracking Tool: second edition. Gland, Switzerland: World Bank/WWF Forest Alliance, 2007. 21p.

THIOLLENT, Michel. Crítica metodológica, investigação social e enquete operária. São Paulo: Polis, 1982. $270 \mathrm{p}$.

UNEP-WCMC; IUCN. Protected Planet Report 2016. Cambridge UK and Gland, Switzerland: UNEPWCMC and IUCN, 2016.

YAMAMOTO, J. K.; LANDIM, P. M. B. Geoestatística: Conceitos e Aplicações. São Paulo: Editora Oficina de Textos, 1.ed., 2013, 215p.

UNEP-WCMC, IUCN and NGS Protected Planet Report 2018. UNEP-WCMC, IUCN and NGS: Cambridge UK; Gland, Switzerland; and Washington, D.C., USA. (2018). 70p.

WWF Brasil - World Wide Found for Nature. Áreas Protegidas ou Espaços Ameaçados: O Grau de Implementação e a vulnerabilidade das Unidades de Conservação federais Brasileiras de Uso Indireto. Org. DE SÁ, Rosa M. Lemos; FERREIRA, Leandro. Brasília: WWF, 1999. 32p.

WWF Brasil - World Wide Found for Nature. Avaliação da gestão das unidades de conservação: métodos RAPPAM (2015) e SAMGE (2016). Brasília: WWF Brasil, $1^{a}$ ED., 2017, 127p. 